\title{
ANAESTHETIC MANAGEMENT OF AN ADULT PATIENT WITH UNCORRECTED VENTRICULAR SEPTAL DEFECT POSTED FOR OBSTRUCTED INGUINAL HERNIA REPAIR
}

Shivaramu B. T11, Shashank M. R², Prajwal Patel H. S ${ }^{3}$

\section{HOW TO CITE THIS ARTICLE:}

Shivaramu B. T, Shashank M. R, Prajwal Patel H. S. "Anaesthetic Management of an Adult Patient with Uncorrected Ventricular Septal Defect Posted for Obstructed Inguinal Hernia Repair". Journal of Evolution of Medical and Dental Sciences 2015; Vol. 4, Issue 66, August 17; Page: 11574-11579,

DOI: $10.14260 /$ jemds/2015/1671

\begin{abstract}
Adults with unrepaired acyanotic heart disease scheduled for a non-cardiac surgery present serious concerns for the anaesthesiologists. Ventricular septal defect (VSD), is an acyanotic congenital heart disease, characterized by a left to right shunt. The incidence varies between 2 to 6 per 1000 live births. It is found in $30 \%$ to $60 \%$ of all the newborns with congenital heart disease, thus making it one of the most common congenital heart diseases VSD is the commonest form of CHD. Unlike ASD, the majority will undergo spontaneous closure: $40 \%$ by the age of $2 \mathrm{yr}$ and $90 \%$ by the age of $10 \mathrm{yr}^{1}{ }^{1}$ The goal of anesthetic management should be to maintain intravascular volume. Systemic and pulmonary vascular resistance changes, such as might occur due to acidosis, hypothermia, hypercarbia or excessive airway pressures, should be avoided. Maintenance of preload, contractility and sinus rhythm is of major importance. The complex pathophysiologies of such heart disease, in addition to the circumstances of emergency operation, exacerbate the total anesthetic risk. We present here a rare case of obstructed right sided inguinal hernia repair with successful outcome in an adult with ventricular septal defect.
\end{abstract}

KEYWORDS: Ventricular septal defect, Obstructed inguinal hernia, Epidural anaesthesia

INTRODUCTION: Congenital heart disease (CHD) complicates $\sim 1 \%$ of all live births in the general population-about 40,000 births/year-but occurs more frequently in the offspring (About 4-5\%) of women with CHD. Congenital heart diseases (CHD), can be either cyanotic or acyanotic, complicate approximately $1 \%$ of all the live births in the general population, and $4 \%$ of off springs of women with CHD. ${ }^{1}$ Palliative and corrective surgeries, now being done commonly at a younger age has improved the survival of such patients. The anatomic and physiologic changes in the heart and circulation due to any specific CHD lesion are not static but, rather, progress from prenatal life to adulthood. Malformations that are benign or escape detection in childhood may become clinically significant in the adult. Substantial number of affected infants and children reach adulthood because of successful medical and surgical management, ${ }^{2}$ or because they have successfully adapted to their particular cardiovascular physiology. The anaesthesiologist will therefore frequently encounter patients with congenital cardiac problems presenting for noncardiac surgery. ${ }^{3}$ The cyanotic CHD present early in childhood, but acyanotic CHD may be relatively asymptomatic till later in life, due to balance between the systemic and pulmonary circulation. ${ }^{4}$ A VSD is one of the most common of all cardiac birth defects, either as isolated defects or as a component of a combination of anomalies. The VSD is usually single and situated in the membranous or midmuscular portion of the septum. The functional disturbance depends on its size and on the status of the pulmonary vascular bed. Only small- or moderate-size VSDs are seen initially in adulthood, as most patients with an isolated large VSD come to medical or surgical attention early in life Ventricular septal defect (VSD), one of the 
commonest acyanotic heart diseases, can occur as an isolated defect or as a component of a combination of anomalies. Usually, isolated small or moderate sized defects are initially detected in adulthood, and can present with significant perioperative morbidity and mortality, if not evaluated carefully and managed meticulously.

We report a case of a 28 years old male with VSD with obstructed right sided inguinal hernia, posted for emergency hernia repair successfully managed with sole epidural anaesthesia.

CASE SUMMARY: A 28 years old male patient, weighing 64kgs, with VSD, was posted for emergency obstructed right sided inguinal hernia repair. Since childhood patient had recurrent episodes of breathlessness on exertion (NYHA grade-2) with palpitation and recurrent episodes of respiratory tract infections. There was a history of one episode of syncopal attack, exertional breathlessness (NYHA grade II) and easy fatigability for which he consulted a physician, was investigated and subsequently was diagnosed to be suffering from a ventricular septal defect. The patient also gave history of chest pain on exertion- walking more than half a kilometer or doing moderate worksuggesting poor exercise tolerance. There was no history of orthopnoea or paroxysmal nocturnal dyspnoea or palpitations. He was under regular treatment with injection benzathine penicillin 1.2G every 3 weeks. Personal and family histories were also insignificant. On examination, the patient was thin build with height of $158 \mathrm{~cm}$ and weight 64kgs. Lying in left lateral position he had pulse rate 76 per minute, blood pressure $110 / 70 \mathrm{~mm} \mathrm{Hg}$, respiratory rate 20 per minute and oxygen saturation was $95 \%$ in room air and $100 \%$ with oxygen flow of 6 liters per minute by face mask. The jugular venous pressure and carotid pulse were normal.

There was no pallor, clubbing, pedal edema, icterus, lymphadenopathy or cyanosis and chest was bilaterally clear. The cardiac examination demonstrated no heave or thrill. On auscultation there was a grade III pansystolic murmur, in the left lower sternal area with no signs of pulmonary hypertension, congestive cardiac failure or infective endocarditis. The patient's respiratory and neurological examinations showed normal results and airway examination showed mallampatti grade II with adequate mouth opening. Electrocardiogram showed poor progression of ' $R$ ' wave in V1-V3 leads with mild left axis deviation. Chest x-ray showed cardiac enlargement. In concurrence with the clinical findings, the 2D- ECHO showed a $6 \mathrm{~mm}$ restrictive sub aortic VSD, with left to right shunt and pressure gradient of $30 \mathrm{~mm} \mathrm{Hg}$ with preserved left ventricular systolic function (LVEF$60 \%$ ). There was dilatation of right ventricle and other valves were normal and there was no evidence of associated atrial septal defect, patent ductus arteriosus, coarctation of aorta or other congenital anomalies. Preoperative investigations of the patient, including a hemogram, renal and liver function tests, coagulation profile and ultrasonography of the kidney-ureter-bladder, were within normal limits.

A thorough cockpit drill was performed. All emergency drugs like adrenaline, noradrenaline, dopamine, dobutamine, nitroglycerine, amiodarone were kept ready and arrangements were made for emergency defibrillation along with other resuscitation equipments.

Since, the case was emergency patient was not kept Nil per Orally. Precautions such as Inj. Metaclopromaide $10 \mathrm{mg}$ i.v. and Inj. Ranitidine 50mg i.v. given. A $18 \mathrm{G}$ intravenous cannula was inserted and secured in left forearm vein. The baseline parameters were pulse rate: 70/min, BP: 110/76 mm Hg and $\mathrm{Spo}_{2}$ 95\% in room air and $\mathrm{Spo}_{2}$ 100\% on oxygen by facemask. Ampicillin1gm and gentamicin80mg were administered intravenously before anesthesia for prophylaxis against bacterial endocarditis. 
With patient in left lateral position, epidural needle 18G was inserted in L3-4 interspace observing aseptic precautions after local infiltration of skin with $2 \%$ lignocaine. Epidural space was located with loss of resistance to saline technique. Skin to epidural space distance was $3 \mathrm{~cm}$. Careful aspiration was done through epidural catheter. The epidural location of the catheter was established with a test dose of $3 \mathrm{ml}$ of $2 \%$ lidocaine with epinephrine 1: 200000 . Then $8 \mathrm{ml}$ of $0.25 \%$ bupivacaine with fentanyl $25 \mathrm{mcg}$ was administered epidurally. A dose of $4 \mathrm{ml}$ was repeated after 45 minutes. The patient remained haemodynamically stable. $\mathrm{SpO}_{2}$ maintained between $96 \%$ and $100 \%$. Throughout the intraoperative period, a close watch was kept on the vital parameters. Procedure lasted for $1 \mathrm{hr}$ $30 \mathrm{mins}$ amount of intravenous fluid used was $1200 \mathrm{ml}$. Amount of blood loss was $150 \mathrm{ml}$. After surgery, the patient was shifted to post anesthesia care unit for continuous hemodynamic and $\mathrm{SpO}_{2}$ monitoring with oxygen via a face mask. After 6 hours he was shifted from PACU to HDU. Subsequent recovery was uneventful and satisfactory. Inj. Bupivacaine $0.125 \%-10 \mathrm{ml}$ was administered twice daily for epidural analgesia. Further postoperative period was uneventful and subsequently, the patient was discharged home.

DISCUSSION: A review of the literature yields several case reports of adults with unrepaired congenital heart disease undergoing anesthesia for non-cardiac surgery. One account describes the anesthetic care of a 24-year-old female patient with unrepaired tetralogy of Fallot undergoing laparoscopic cholecystectomy for pancreatitis5. Although previously palliated with a modified Blalock-Taussig (Right subclavian to right pulmonary artery) shunt, her tetralogy of Fallot remained unrepaired in terms of the essential components. A different case study reviews the successful anesthetic management of a 22-year-old woman undergoing appendectomy. She suffered from situs solitus, a severe cyanotic heart defect consisting of pulmonary atresia, overriding aorta, concordant atrioventricular shunts, aortopulmonary collateral circulation, and a large ventricular septal defect (VSD).6 Both a 22-year old man who received anesthesia for a repeated craniotomy to repair a chronic brain abscess and a 50-year-old woman anesthetized for emergency laparotomy presented with complex cyanotic congenital heart disease, further complicated by the presence of Eisenmenger syndrome. ${ }^{7,8}$

The exact pathophysiology of the disease should be understood by the anesthetist before choosing the appropriate anaesthetic plan. In these conditions, the challenge surrounds the management of the systemic and, especially, of the pulmonary vascular resistances. Their increase or decrease may, respectively, augment right ventricular pressure/volume loads or reduce pulmonary blood flow. Thus, the maintenance of pulmonary vascular resistance, right ventricular preload and contractility, and left ventricular afterload is of great importance.1-3 At the same time, hypothermia, hypercarbia, hypoxia, acidosis, excessive airway pressures and the use of adrenergic agents, such as ephedrine and norepinephrine, should be avoided. Anatomically, approximately $70 \%$ of these defects are located in the membranous portion of the intraventricular septum, $20 \%$ in the muscular portion of the septum, 5\% just below the aortic valve causing aortic regurgitation, and $5 \%$ near the junction of the mitral and tricuspid valve (Atrioventricular canal defect).

The physiologic significance of a VSD depends on the size of the defect and the relative resistance in the systemic and pulmonary circulations. If the defect is small, there is minimal functional disturbance as pulmonary blood flow is only modestly increased. If the defect is large, the ventricular systolic pressures equalize and the magnitude of systemic and pulmonary blood flow is determined by the relative vascular resistances of these two circulations. 
Initially, systemic vascular resistance exceeds pulmonary vascular resistance, and left-to-right intracardiac shunting predominates. Over time, the pulmonary vascular resistance increases, and the magnitude of the left-to right intracardiac shunting decreases; eventually, the shunt may become right to left with the development of arterial hypoxemia (Cyanosis) leading to Eisenmenger's syndrome. The natural history of VSDs in adults depends largely on their size and associated shunt flow. Patients with a VSD that has closed spontaneously and normal ventricular function have a normal life expectancy. Patients with a small restrictive VSD who have a normal PVR have an excellent prognosis, ${ }^{4}$ albeit with a considerable risk of endocarditis. ${ }^{5}$ With a small VSD, it is unlikely that PVD will develop in later life. The American Heart Association significantly changed its guidelines for prevention of infective endocarditis (IE) in 2007, resulting in a narrowing of the indications for administering IE prophylaxis. The patient must have BOTH a cardiac indication, and a surgical/procedural indication.

The major cardiac indications are: 1 . prosthetic cardiac valve or material; 2. previous IE; 3. congenital heart disease, but ONLY a) unrepaired cyanotic CHD including palliative shunts and conduits; b) completely repaired CHD with prosthetic material or device during the first 6 months after the procedure; c) repaired CHD with residual defects at or near the site of a prosthetic patch or device; 4. Cardiac transplant recipients who develop valvulopathy. The surgical/procedural indications are 1. All dental procedures that involve manipulation or the gingival tissue or perforation of the oral mucosa; 2. Respiratory tract procedures or procedures on infected skin, or musculoskeletal tissue. IE prophylaxis is not recommended for simple gastrointestinal or genitourinary procedures where the mucosa is not incised, i.e. simple endoscopy and cystoureteroscopy, but is recommended for surgery where mucosa is incised. ${ }^{9}$ Patients who have had pulmonary hypertension, significant problems can develop in the perioperative period even if they are undergoing non-cardiac surgery ${ }^{10}$. The aim of the management of these patients is to avoid factors that predispose to pulmonary hypertension and to reverse any reversible factors like acidosis, hypercapnia, hypoxia etc.11-13 Therefore, we opted for regional anesthesia in our patient, because of the risk of alteration in pulmonary blood flow.

Major threat with use of regional anesthesia is reduction in systemic vascular resistance and reversal of left to right shunt, which may results in hypoxia. When anesthetizing patients with congenital heart disease, under either a regional technique or general anesthesia, the following factors must be kept in mind; prevention of accidental intravenous infusion of air bubbles by using loss of resistance to saline rather than air to identify the epidural space, ${ }^{9}$ a slow onset of epidural analgesia is useful, as rapid fall in SVR could result in reversal of shunt.14-16 Supplemental $\mathrm{O}_{2}$ should be given to the patient throughout the procedure if regional technique is used. It is advisable to monitor $\mathrm{O}_{2}$ saturation as mild hypoxaemia may result in an increase in PVR and reversal of shunt flow. We successfully treated the fall in SVR by using injection phenylephrine, which also prevents any reversal of left to right shunt and or any hypoxaemia.17,18 


\section{REFERENCES:}

1. Perloff JK. Survial patterns without cardiac surgery or interventional catheterization: a narrowing base. In: Perloff JK, Childs JS, eds. Congenital Heart Disease in Adults, 2nd Edn. Philadelphia: WB Saunders, 1998; 15 \pm 53 .

2. Child J S, Aboulhosn J. Congenital heart disease in adult. In Longo D L, Kasper D L, Jameson J L, Fauci A S, Hauser S L, Loscalzo J (Ed). Harrison's principles of internal medicine. $18^{\text {th }}$ edition. Volume 2. United States of America: Mc Graw Hill; 2012.1920-8.

3. Abraham B, Shivanna S, Tejesh C A. Dextrocardia and ventricular septal defect with situs inversus: Anesthetic implications and management. Anesthesia: Essays and Researches 2012; 6(2): 207-9.

4. Magee AG, Fenn L, Vellekoop J, et al. Left ventricular function in adolescents and adults with restricive ventricular septal defect and moderate left-to-right shunting. Cardiol Young 2000; 10: $126 \pm 9$.

5. Andrews JD. Laparoscopic cholecystectomy for the adult with unrepaired tetralogy of Fallot: a case report. AANA J. 1999; 67(1): 67-71.

6. Chatzidaki R, Koraki E, Vasiliadis K, Aslanidis T, Vasilakos D. Appendectomy for an adult with cyanotic congenital heart disease. Minerva Anestesiol. 2009; 75(4): 225-228.

7. Asamoto M, Kitamura T, Ohno N, et al. Anesthetic management for emergency laparotomy in an adult patient with Eisenmenger syndrome: a case report [abstract, Japanese]. Masui. 2009; 58(8): 1021- 1024.

8. Kakemizu M, Sakai H, Suzuki K, Okazaki A. Anesthetic management of craniotomy for a young adult patient with congenital cyanotic heart disease [abstract, Japanese]. Masui. 2010; 59(2): 202-205.

9. Warnes CA, Williams RG, Bashore TM, et al. ACC/AHA 2008 guidelines for the management of adults with congenital heart disease: a report of the American College of Cardiology/American Heart Association Task Force on Practice Guidelines. J Am Coll Cardiol. 2008; 52: e1-121.

10. Neumayer U, Stone S, Somerville J. Small ventricular septal defects in adults. Eur Heart J 1998; 19: $1573 \pm 82$.

11. Mohindra R, Beebe D S, Belani K G. Anaesthetic Management of Patients with Congenital Heart Disease Presenting for Non-Cardiac Surgery. Annals of Cardiac Anaesthesia 2002; 5: 15-24.

12. Sharma A, Parasa S, Gudivada K, Gopinath R. Differential cyanosis and undiagnosed eisenmenger's syndrome: The importance of pulse oximetry. Anesthesia: Essays and Researches 2014; 8(2): 233-5.

13. Stayer S. Anesthesia for the Patient with Congenital Heart Disease Undergoing Non-cardiac Surgery. Patient with CHD for Non-cardiac Surgery. SPA Refresher Course April 2010; 1-10.

14. UD Allen. Infective endocarditis: updated guidelines, Canadian paediatric society, Infectious diseases and immunization committee, Paediatric child health 2010; 15(4): 205-8.

15. K Sandhya, Shivanna S, C A Tejesh, N Rathna. Labour analgesia and anaesthetic management of a primigravida with uncorrected pentology of fallot. Indian Journal of Anaesthesia 2012; 56(2): 186-8.

16. Solanki S, Jain A, Singh A, Sharma A. low dose sequential combined spinal epidural anesthesia for cesarean section in patient with uncorrected tetralogy of fallot. Saudi journal of anaesthesia 2011; 5(3): 320-2. 


\section{CASE REPORT}

17. Andropoulos D B. Anesthesia for the Patient with Congenital Heart Disease For Noncardiac Surgery. American society of anesthesiologists: anesthesiology 2011; 206: 1-9.

18. Cannesson M, Earing M G, Collange V, Kersten J R. Anesthesia for Noncardiac Surgery in Adults with Congenital Heart Disease. Anesthesiology 2009; 111: 432-40.

\section{AUTHORS:}

1. Shivaramu B. T.

2. Shashank M. R.

3. Prajwal Patel H. S.

\section{PARTICULARS OF CONTRIBUTORS:}

1. Assistant Professor, Department of Anaesthesia, AIMS, B. G. Nagar, Bellur.

2. Assistant Professor, Department of Anaesthesia, AIMS, B. G. Nagar, Bellur.

3. Assistant Professor, Department of Anaesthesia, AIMS, B. G. Nagar, Bellur.

NAME ADDRESS EMAIL ID OF THE CORRESPONDING AUTHOR:

Dr. Shivaramu B. T, \#1520, 2 ${ }^{\text {nd }}$ Main, $7^{\text {th }}$ cross,

Vivekananda Nagara,

Mysore- 570023.

E-mail: shivarambidrakote@gmail.com

Date of Submission: 28/07/2015. Date of Peer Review: 29/07/2015. Date of Acceptance: 10/08/2015. Date of Publishing: 17/08/2015.

\section{COMPETING INTERESTS: None}

